\title{
Supercorrelated Radiance in Nonlinear Photonic Waveguides
}

\author{
Zhihai Wang $\odot,{ }^{1, *}$ Tuomas Jaako $\odot,{ }^{2}$ Peter Kirton $\odot,{ }^{2, \dagger}$ and Peter Rabl $\odot^{2}$ \\ ${ }^{1}$ Center for Quantum Sciences and School of Physics, Northeast Normal University, Changchun 130024, China \\ ${ }^{2}$ Vienna Center for Quantum Science and Technology, Atominstitut, TU Wien, 1040 Vienna, Austria
}

(Received 9 December 2019; accepted 6 May 2020; published 27 May 2020)

\begin{abstract}
We study the collective decay of two-level emitters coupled to a nonlinear waveguide, for example, a nanophotonic lattice or a superconducting resonator array with strong photon-photon interactions. Under these conditions, a new decay channel into bound photon pairs emerges, through which spatial correlations between emitters are established by regular interference as well as interactions between the photons. We derive an effective Markovian theory to model the resulting decay dynamics of an arbitrary distribution of emitters and identify collective effects beyond the usual phenomena of super- and subradiance. Specifically, in the limit of many close-by emitters, we find that the system undergoes a supercorrelated decay process where all the emitters are either in the excited state or in the ground state but not in any of the intermediate states. The predicted effects can be probed in state-of-the-art waveguide QED experiments and provide a striking example of how the dynamics of open quantum systems can be modified by many-body effects in a nonharmonic environment.
\end{abstract}

DOI: 10.1103/PhysRevLett.124.213601

The radiative decay of an excited atom, induced by its coupling to the continuum of electromagnetic modes, is a prototypical example of irreversible energy loss in quantum systems. Dicke [1,2] showed that this process can be modified significantly in settings with multiple closely spaced emitters, where the decay rate can be collectively enhanced or suppressed due to interference. Recently, such super- and subradiant effects have gained considerable attention in the context of waveguide QED [3-11], where atoms [12-15], quantum dots [16], or superconducting qubits [17-21] are coupled to nanophotonic or microwave waveguides. Along with enhancing the rate of decay, the strong transverse mode confinement in such structures also leads to strongly correlated emission between distant emitters. Under such conditions, collective radiation effects can give rise to self-organization [22,23], longrange entanglement [24-29], and efficient light-matter interfaces [30,31].

Collective radiance is usually modeled under the premise that the environment is represented by independent harmonic oscillators. However, in nanophotonic lattices, plasmonic waveguides, and superconducting resonator arrays, intrinsic or engineered nonlinearities [32-39] can become significant at the level of a few photons, breaking the validity of this assumption. Therefore, a natural question arises: how radiation behaves in a strongly interacting environment. In this Letter, we address this question by analyzing the decay of multiple two-level systems (TLSs) into an array of coupled cavities with strong on-site photon-photon interactions. Specifically, we focus on emitter frequencies below the edge of the propagation band, where singlephoton emission is suppressed and an interaction-induced decay channel, which forces the TLSs to emit photons in bound pairs, dominates. In this regime, the decay dynamics is determined by a new correlation length related to the size of attractively bound photon pairs. These correlations give rise to collectively enhanced and suppressed decay processes beyond the effects of super- and subradiance in linear photonic systems. Most remarkably, for many closely spaced emitters, we find a collective acceleration beyond the $N^{2}$ scaling of superradiance. This has the intriguing consequence that, at any time, almost all TLSs are found in either the excited or the ground state but not in any of the intermediate mixed configurations. In this limit, spontaneous emission becomes perfectly correlated.

Model.-We consider a system of $N$ TLSs with ground state $|g\rangle$ and excited state $|e\rangle$, which interact with a onedimensional (1D) array of tunnel-coupled cavities, as schematically shown in Fig. 1(a). The photonic lattice is modeled by a tight-binding Hamiltonian $(\hbar=1)$

$H_{\mathrm{ph}}=\sum_{n} \omega_{c} a_{n}^{\dagger} a_{n}-\frac{U}{2} a_{n}^{\dagger} a_{n}^{\dagger} a_{n} a_{n}-J\left(a_{n}^{\dagger} a_{n+1}+\right.$ H.c. $)$,

where $a_{n}$ is the photon annihilation operator on site $n$ and $\omega_{c}$ and $4 J>0$ are the central frequency and the total width of the propagation band, respectively. The second term in Eq. (1) accounts for a Kerr-like interaction between the photons, which we assume to be attractive, i.e., $U>0$. The Hamiltonian for the whole system is

$$
H=H_{\mathrm{ph}}+\frac{\omega_{e}}{2} \sum_{i=1}^{N} \sigma_{i}^{z}+g \sum_{i=1}^{N}\left(a_{n_{i}} \sigma_{i}^{+}+a_{n_{i}}^{\dagger} \sigma_{i}^{-}\right),
$$


(a)
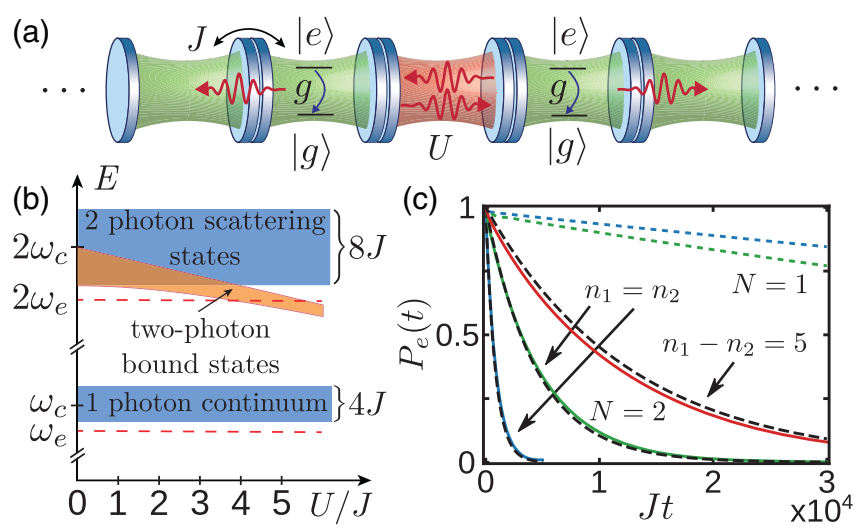

FIG. 1. (a) Sketch of the waveguide QED setup, where multiple TLSs are coupled to a photonic lattice with nearest-neighbor tunneling $J$ and on-site interaction $U$. (b) The corresponding band structure in the one- and two-photon subspace. (c) Evolution of the excited state population $P_{e}(t)$ for $N=1$ (dotted curves) and $N=2$ (solid curves) emitters with frequency $\omega_{e}<\omega_{c}-2 J$, as indicated in (b). The parameters for the three solid curves are $U=J, K_{0} \approx 0.1 \pi, g / J=0.02,\left(\omega_{e}-\omega_{c}\right) / J=-2.04$, and $\left|n_{1}-n_{2}\right|=0(5)$ for the green (red) line and $U=4 J, K_{0} \approx 0.5 \pi$, $g / J=0.1$, and $\left(\omega_{e}-\omega_{c}\right) / J=-2.45$ for the blue line. For all plots, $\kappa / J=3 \times 10^{-4}$, and the dashed lines show the approximate analytic result of Eq. (7).

where the $\sigma_{i}^{ \pm, z}$ are the usual Pauli operators for the $i$ th TLS located at lattice site $n_{i}, \omega_{e}$ is the TLS transition frequency, and $g$ is the coupling strength. For small $g$ and $\omega_{e} \in\left[\omega_{c}-2 J, \omega_{c}+2 J\right]$, an excited TLS can decay with a characteristic rate $\Gamma_{1} \sim g^{2} / J$ into a propagating singlephoton wave packet. For multiple TLSs, the emitted photons can interfere, which gives rise to the well-studied effects of super- and subradiance [1,2,31,40-49].

In the following, we are interested in a scenario where $\omega_{e}<\omega_{c}-2 J$ lies below the propagation band, such that this regular decay channel is absent. As indicated in Fig. 1(b), under this condition it is still possible for two or more emitters to decay via a resonant excitation of a bound two-photon state. For a lattice of $N_{c} \gg 1$ cavities, and neglecting the emitters now, a general two-photon eigenstate can be written as $\left|\Psi_{K}\right\rangle=(1 / \sqrt{2}) \sum_{n, m} \Psi_{K}(n, m) a_{n}^{\dagger} a_{m}^{\dagger}|\mathrm{vac}\rangle$, where $|v a c\rangle$ is the vacuum state of the waveguide and the wave function $\Psi_{K}(n, m)=e^{i K(n+m) / 2} \psi_{K}(n-m) / \sqrt{N_{c}}$ is symmetric and can be decomposed into center-of-mass and relative components. For each $K \in(-\pi, \pi]$, there is a band of scattering states $\psi_{K}^{q}(r) \sim \cos \left(q r-\varphi_{K}\right)$, which extend across the whole lattice and have energies $E_{K}^{q}=2 \omega_{c}-4 J_{K} \cos (q)$, where $J_{K}=J \cos (K / 2)$. In addition, there exists one bound state per $K$ with energy $E_{K}^{b}=2 \omega_{c}-\sqrt{U^{2}+16 J_{K}^{2}}[50,52,53]$ and an exponentially localized wave function $\psi_{K}^{b}(r) \propto e^{-|r| / \lambda_{K}}$, with size $\lambda_{K}^{-1}=\operatorname{asinh}\left(\mathcal{U}_{K}\right)$, where $\mathcal{U}_{K}=U /\left(4 J_{K}\right)$. As shown in Fig. 1(b), the energies $E_{K}^{b}$ largely overlap with the scattering states for small $U$, but for $U \gtrsim J$ a finite band of propagating two-photon states appears below the scattering continuum. The repulsively bound counterparts of these states have been observed with cold atoms in optical lattices [54], and they also exist in 2D and 3D lattices, although at slightly larger interactions [50].

Correlated two-photon decay.-In Fig. 1(c), we show the evolution of the excited state population, $P_{e}(t)=$ $\sum_{i}\left\langle\sigma_{i}^{+} \sigma_{i}^{-}\right\rangle / N$, for both one and two initially excited TLSs with a frequency below the band edge and including a small loss rate $\kappa$ for each cavity. For a single TLS, we observe only a small residual decay of about $\Gamma_{1} \approx \kappa g^{2} /\left(4 \sqrt{J \delta_{0}^{3}}\right)[50,55]$, where $\delta_{0}=\omega_{c}-\omega_{e}-2 J$ is the detuning from the band edge. However, two nearby TLSs decay at a much faster rate, which is approximately independent of $\kappa$. To understand this behavior, we consider the weak-coupling limit $g \ll J, U$ and write the wave function of the system as

$$
\begin{aligned}
\left|\phi_{2}\right\rangle(t)= & e^{-2 i \omega_{e} t}\left\{c_{e}(t) \sigma_{1}^{+} \sigma_{2}^{+}+\sum_{K} c_{K}(t) B_{K}^{\dagger}\right. \\
& \left.+\sum_{k}\left[c_{1 k}(t) \sigma_{1}^{+}+c_{2 k}(t) \sigma_{2}^{+}\right] a_{k}^{\dagger}\right\}|g, g, \mathrm{vac}\rangle
\end{aligned}
$$

where $a_{k}^{\dagger}=\sum_{n} e^{i k n} a_{n}^{\dagger} / \sqrt{N_{c}}$ and $B_{K}^{\dagger}$ is the creation operator for a bound photon pair, $\left|\Psi_{K}^{b}\right\rangle=B_{K}^{\dagger}|\mathrm{vac}\rangle$. This does not include the two-photon scattering states, which play a negligible role in the dynamics [50]. Since the one-photon states $\left|i, 1_{k}\right\rangle=\sigma_{i}^{+} a_{k}^{\dagger} \mid g, g$, vac $\rangle$ are separated by an energy gap $\delta_{k}=\omega_{c}-2 J \cos (k)-\omega_{e} \gg g / \sqrt{N_{c}}$, they can be eliminated using perturbation theory. We hence obtain an effective coupling between the TLSs and the continuum of two-photon bound states [50]:

$$
\begin{gathered}
i \dot{c}_{e}=-\frac{g^{2}}{J \sqrt{N_{c}}} \sum_{K} e^{i K\left(n_{1}+n_{2}\right) / 2} f_{K}\left(n_{1}, n_{2}\right) c_{K}, \\
i \dot{c}_{K}=\Delta_{K} c_{K}-\frac{g^{2}}{J \sqrt{N_{c}}} e^{-i K\left(n_{1}+n_{2}\right) / 2} f_{K}\left(n_{1}, n_{2}\right) c_{e},
\end{gathered}
$$

where $\Delta_{K}=E_{K}^{b}-2 \omega_{e}$. Equation (5) is valid only for bound photon states below the propagation band, $\Delta_{K} \approx 0$, which are, however, the relevant modes in the following discussion. The matrix element $f_{K}\left(n_{1}, n_{2}\right) \equiv f_{K}\left(n_{1}-n_{2}\right)$ depends only on the relative separation and can be expressed in terms of the two-photon correlation function:

$$
\begin{aligned}
f_{K}\left(n_{1}, n_{2}\right)= & -i \sqrt{N_{c}} J \int_{0}^{\infty} d \tau e^{i K\left(n_{1}+n_{2}\right) / 2} e^{-i \omega_{e} \tau} \\
& \times\left\langle\operatorname{vac}\left|B_{K}\left[a_{n_{2}}^{\dagger} a_{n_{1}}^{\dagger}(\tau)+a_{n_{1}}^{\dagger} a_{n_{2}}^{\dagger}(\tau)\right]\right| \mathrm{vac}\right\rangle .
\end{aligned}
$$

As illustrated in Fig. 2(a), this quantity can be interpreted as follows: The first TLS emits a virtual photon at $n_{1}$. 

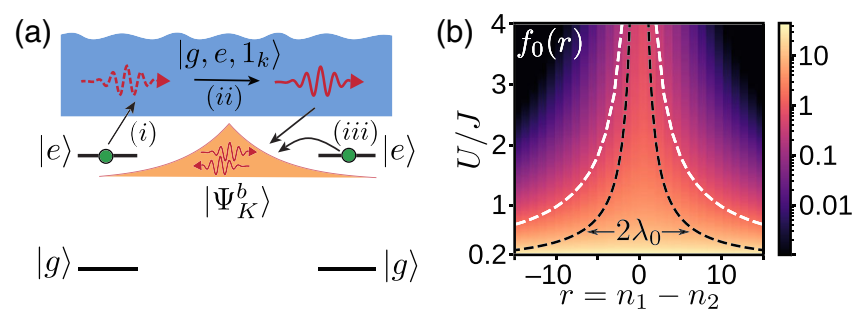

FIG. 2. (a) Illustration of the correlated two-photon decay process. The first TLS emits a virtual photon (i), which propagates for a time $\sim 1 / \delta_{k}$ (ii) before it combines with the second emitted photon into a propagating bound state (iii). (b) Contour plot of $f_{K=0}\left(n_{1}, n_{2}\right)$ for different ratios $U / J$. The black and the white dashed lines represent the bound-state size $\lambda_{K=0}$ and the $1 / e$ decay length of $f_{0}(r)$, respectively.

This photon propagates for a time $\tau$ before another photon is created by the second emitter at $n_{2}$. Then $f_{K}\left(n_{1}, n_{2}\right)$ is the overlap of this photon pair (and its symmetric counterpart) with the two-photon bound state $\left|\Psi_{K}^{b}\right\rangle$. Therefore, as shown in Fig. 2(b), the correlations induced can exceed the size of the two-photon bound state $\lambda_{K}$ and depend on propagation and interference effects of the intermediate single-photon states.

To proceed, we assume that the energy of the emitters lies within the band of bound two-photon states, $2 \omega_{e} \in$ $\left[E_{0}^{b}, E_{\pi}^{b}\right]$, and eliminate the dynamics of those states using a Wigner-Weisskopf approximation. This is valid when the group velocity of the emitted photons, $v_{g}\left(K_{0}\right)=$ $\partial E_{K}^{b} / \partial K_{K=K_{0}}$, where $K_{0}$ is determined by $2 \omega_{e}=E_{K_{0}}^{b}$, is larger than the effective coupling $\sim g^{2} / J$. In this case, we obtain an exponential decay of the doubly excited state, $P_{e}(t)=e^{-\Gamma t}$, with a rate [50]

$$
\Gamma=\frac{2 g^{4}}{J^{3}}\left|f_{K_{0}}\left(n_{1}, n_{2}\right)\right|^{2} \tilde{\rho}\left(K_{0}\right),
$$

where $\tilde{\rho}(K)=J / v_{g}(K)$ is the normalized density of bound two-photon states and $v_{g}(K)=4 J^{2} \sin (K) /$ $\sqrt{U^{2}+16 J^{2} \cos ^{2}(K / 2)}$. Figure 1(c) and additional examples in Ref. [50] show that this approximate result agrees very well with exact numerical simulations for typical rates in the range of $\Gamma / J \sim 10^{-4}-10^{-2}$.

Collective radiance.-To analyze the decay of an arbitrary distribution of emitters, we generalize the elimination of the photons from above, deriving a master equation (ME) [56] for the reduced density operator $\rho$ of the TLSs. In a frame rotating with $\omega_{e}$, this equation is [50]

$$
\dot{\rho}=-i\left(H_{\mathrm{eff}} \rho-\rho H_{\mathrm{eff}}^{\dagger}\right)+\mathcal{J}(\rho),
$$

where $\mathcal{J}(\rho)=\sum_{i, j, k, l} \Gamma_{i j, k l} \sigma_{i}^{-} \sigma_{j}^{-} \rho \sigma_{k}^{+} \sigma_{l}^{+}$is the recycling term with $\Gamma_{i j, k l}=\Gamma_{0} \operatorname{Re}\left\{A_{i j, k l}\right\}$ and $\Gamma_{0}=2 g^{4} \tilde{\rho}\left(K_{0}\right) / J^{3}$ and we have introduced the non-Hermitian Hamiltonian

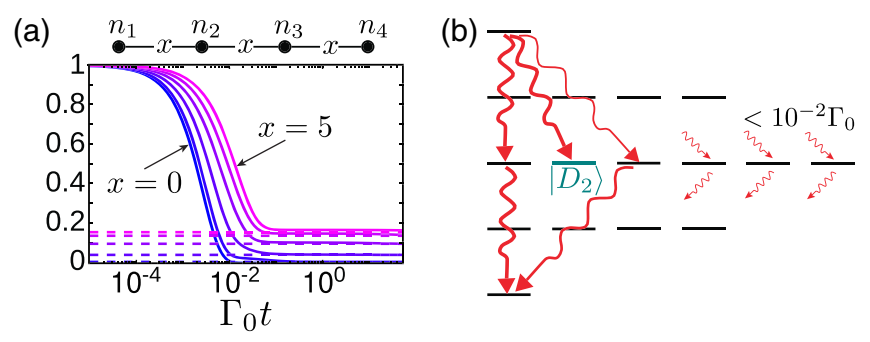

FIG. 3. (a) The dynamics of $P_{e}(t)$, obtained from Eq. (8), for $N=4$ TLSs with different spacings, $n_{i+1}-n_{i}=x$. The solid lines represent the numerical results, and the dashed lines are from a semianalytic calculation [50]. The parameters are $U=J$, $K_{0} \approx 0.1 \pi, g / J=0.02$, and $\left(\omega_{e}-\omega_{c}\right) / J=-2.04$. (b) Sketch of the dominant decay paths into and out of the two-excitation eigenstates of $H_{\text {eff }}$. The thickness of the lines indicates the relative strength of the decay rates [50].

$H_{\text {eff }}=-i \frac{\Gamma_{0}}{2} \sum_{i, j, k, l} A_{i j, k l} \sigma_{k}^{+} \sigma_{l}^{+} \sigma_{i}^{-} \sigma_{j}^{-}+A_{i j, k l}^{*} \sigma_{i}^{+} \sigma_{j}^{+} \sigma_{k}^{-} \sigma_{l}^{-}$,

which describes collective interactions $\sim \operatorname{Im}\left\{A_{i j, k l}\right\}$ and dissipation processes $\sim \operatorname{Re}\left\{A_{i j, k l}\right\}$, involving up to four TLSs. The form of the amplitudes

$A_{i j, k l}=f_{K_{0}}\left(n_{i}, n_{j}\right) f_{K_{0}}\left(n_{k}, n_{l}\right) e^{i K_{0}\left|\left(n_{k}+n_{l}\right)-\left(n_{i}+n_{j}\right)\right| / 2}$

shows that the radiation-induced correlations depend on two processes. First, correlations with a length scale determined by $f_{K_{0}}$ arise from the nonlinear decay mechanism, as discussed above. Second, photons emitted from different pairs can interfere, which is taken into account by the exponential phase factor. Similar to collective emission in regular waveguides $[55,57,58]$, these interference effects are infinite in range, but here they crucially depend on the relative positions of all the TLSs involved.

Subradiance.-The coherent and dissipative four-body interactions in $H_{\text {eff }}$ make the decay process of a multiemitter system rather complex and can lead to a speed-up of emission as well as the appearance of subradiance, i.e., weakly or even nondecaying states. All single-excitation states, which remain unaffected by two-photon decay, belong to this class of states, but there are additional nontrivial examples. The existence of these states is evident from Fig. 3(a), which shows the dynamics of $N=4$ excited TLSs with different spacings $x$ between them. The ME [Eq. (8)] ensures that only states with an even number of excitations are populated. We observe a fast initial decay on a timescale $\sim 1 /\left[f_{K_{0}}^{2}(x) \Gamma_{0}\right]$, after which the system reaches a quasistationary state with a finite population in the twoexcitation subspace. For equal spacing this is a true stationary state and the excitation remains trapped for all times, while for arbitrary $n_{i}$ it eventually decays but on a much longer timescale.

The fast relaxation into a doubly excited, but nondecaying state is a rather unexpected feature, which is 
explained by Fig. 3(b). Here, the possible decay paths are represented in terms of the eigenstates of $H_{\text {eff }}$ with different excitation numbers. For equal spacing $n_{i+1}-n_{i}=x>0$, we find that in the two-excitation manifold there is one exact dark state, satisfying $H_{\text {eff }}\left|D_{2}\right\rangle=0$, and additional subradiant states with decay rates $\lesssim 10^{-2} \Gamma_{0}$. These other subradiant states are almost decoupled from the waveguide due to symmetry. Therefore, they are long-lived but also hardly populated during the dynamics. In contrast, there is an efficient decay path into state $\left|D_{2}\right\rangle$, which does not decay further. We find that the form of the dark state is

$$
\left.\left.\left|D_{2}\right\rangle=\alpha(x) \mid \text { egge }\right\rangle-\beta(x) \mid \text { geeg }\right\rangle,
$$

where $\alpha(x) / \beta(x)=f_{K_{0}}(x) / f_{K_{0}}(3 x) \geq 1$ [50]. This state is not invariant under the inversion $|g\rangle \leftrightarrow|e\rangle$, which explains why it is possible to have different rates for decaying into and out of it. Similar states also exist for a larger number of emitters, emerging from the combination of long-range interference and the presence of additional correlations, $\sim f_{K_{0}}\left(n_{i}, n_{j}\right)$, which vary considerably across the ensemble.

Collective-spin limit.-From the results of Fig. 3(a), we already see that the rate of emission is enhanced when the spacing between emitters is small. Therefore, we consider next the special case where all TLSs are located in the same lattice site and collective effects are most pronounced. In this limit, the ME reduces to

$$
\dot{\rho}=\frac{\Gamma}{2}\left(2 S_{-}^{2} \rho S_{+}^{2}-\rho S_{+}^{2} S_{-}^{2}-S_{+}^{2} S_{-}^{2} \rho\right),
$$

where $\Gamma=\Gamma_{0} f_{K_{0}}^{2}(0)$ and $S_{-}=\sum_{i} \sigma_{i}^{-}$is the collective spin-lowering operator. Since ME (12) conserves the total spin, we can label all the states involved in the dynamics by their spin projection quantum number $S_{z}|m\rangle=m|m\rangle$, where $|m=N / 2\rangle=\left|e_{1} \ldots e_{N}\right\rangle$ is the fully excited initial state. This leads to a reduced equation for the populations $p_{m}=\langle m|\rho| m\rangle$ :

$$
\dot{p}_{m}=-\Gamma_{m, m-2} p_{m}+\Gamma_{m+2, m} p_{m+2},
$$

where $\Gamma_{m, m-2}=\Gamma\left|\left\langle m-2\left|S_{-}^{2}\right| m\right\rangle\right|^{2}$. In Fig. 4(a), we use this equation to evaluate the collective decay of a large ensemble of TLSs. The nonexponential and accelerated decay is reminiscent of regular Dicke superradiance described by the ME $[2,40]$

$$
\dot{\rho}=\frac{\Gamma}{2}\left(2 S_{-} \rho S_{+}-\rho S_{+} S_{-}-S_{+} S_{-} \rho\right),
$$

but there are important qualitative differences. First, at short times, where $m \approx N / 2$, the decay rate scales as $\Gamma_{m, m-2} \sim N^{2}$. This is $N$ times faster than for $N$ independent TLSs and shows that, even in the initial stage of the evolution, the dynamics is dominated by correlations. For states near the equator of the Bloch sphere, $m \approx 0$, the rates then scale as $\Gamma_{m, m-2} \sim N^{4}$ compared to the $N^{2}$ scaling for
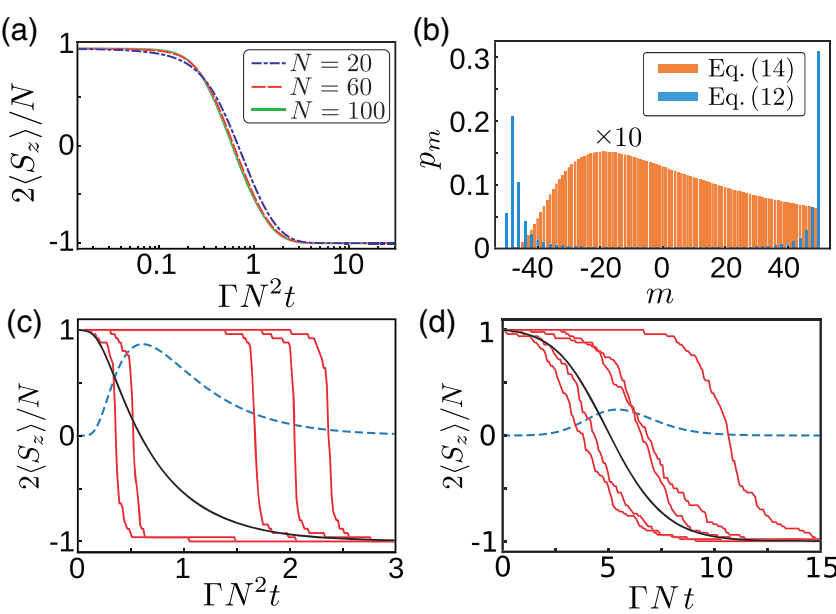

FIG. 4. (a) The evolution of $\left\langle S_{z}\right\rangle$ predicted by Eq. (12) for an initially fully excited ensemble of TLSs. (b) Snapshot of the populations $p_{m}$ for a supercorrelated (blue) and regular superradiant (orange) decay, evaluated when $\left\langle S_{z}\right\rangle=0$. (c),(d) Example trajectories as obtained from a stochastic simulation of Eqs. (12) and (14). The thick lines show the corresponding average values of $2\left\langle S_{z}\right\rangle / N$ (black) and $4\left(\left\langle S_{z}^{2}\right\rangle-\left\langle S_{z}\right\rangle^{2}\right) / N^{2}$ (dashed blue). In (b)-(d), we have used $N=100$.

regular superradiance. Overall, this results in a strongly reduced decay time of $T_{d} \sim 1 /\left(\Gamma N^{2}\right)$.

More importantly, while the dynamics of Eq. (14) can be well described by a mean-field approximation $\left\langle S_{z}^{2}\right\rangle \approx\left\langle S_{z}\right\rangle^{2}$ [40], a similar approach for Eq. (12) fails to accurately capture the system evolution [50]. This can be understood from the snapshot of the populations $p_{m}$ shown in Fig. 4(b), which is taken at the half-decay time $T_{h}$, defined by $\left\langle S_{z}\right\rangle\left(T_{h}\right)=0$. We see that for regular superradiance there is a broad distribution around its mean value. In contrast, the two-photon decay process leads to a bimodal distribution, where most of the population is in the states with $m \approx \pm N / 2$. The intermediate levels are hardly populated, since they decay with a much faster rate. This different behavior can also be clearly seen by looking at individual trajectories of a stochastic ME simulation [59]. The red curves in Figs. 4(c) and 4(d) show example trajectories for the two-photon decay process and regular superradiance, respectively. We see that, in the former case, the time that the system spends near the fully excited state, $T_{e} \sim 1 / N^{2}$, is considerably longer than the time it takes to transition through all the partially excited states, $T_{t} \sim 1 / N^{3}$, such that $T_{t} / T_{e} \sim 1 / N \rightarrow 0$ for large $N$. This means that, when measuring the system at random times during the decay, all TLSs are either still found in the excited state or already in the ground state. It is thus more appropriate to speak of supercorrelated emission rather than just superradiance. This qualitative difference can also be quantified by the correlation parameter

$$
C=\max _{t \in[0, \infty)} \frac{4\left[\left\langle S_{z}^{2}\right\rangle(t)-\left\langle S_{z}\right\rangle^{2}(t)\right]}{N^{2}},
$$


which measures the maximal correlations between the populations of the TLSs during the decay. For independent emitters, $C \approx 0$ in the limit of large $N$, while it reaches a maximal value of $C=1$ when all the emitters are correlated at the point when $\left\langle S_{z}\right\rangle=0$. We find that $C \approx 0.2$ for superradiance and $C \approx 1-O(1 / N)$ [50] for the supercorrelated decay process described by Eq. (12), giving a clear signature of the dynamics discussed above.

Discussion and conclusions. - In summary, we have studied the collective radiance of an ensemble of TLSs coupled to a nonlinear environment. We found that this system supports a strongly correlated decay process outside the scope of conventional super- and subradiance. In the optical domain, implementations of nonlinear photonic lattices have already been proposed for engineering strongly correlated fluids of light [32-36], and similar ideas can be used to explore these decay processes. Alternatively, superconducting qubits can be coupled to an array of microwave resonators, where embedded Josephson junctions provide a strong nonlinearity [60]. In such systems, values of $g \lesssim J, U \approx 50-200 \mathrm{MHz}$ can be achieved with existing technology [61-63]. For $J=100 \mathrm{MHz}$, the achievable decay rates of around $\Gamma \approx 0.1-1 \mathrm{MHz}[50]$ are still fast compared to the bare qubit decay times of $T_{1}=10 \mu \mathrm{s}$ [64]. The supercorrelated limit $N \gg 1$ can further be accessed by replacing the qubits by a large ensemble of Rydberg atoms trapped above the resonator array [65-68] or even above a single nonlinear cavity [50] to further reduce the experimental complexity.

Beyond the specific setting considered in this work, our analysis demonstrates how nontrivial interactions in the environment can strongly modify the qualitative behavior of open quantum systems. In turn, the established relation between collective radiance and few-body effects in the bath can potentially be used as a more general method to probe complex many-body processes through the correlated decay of multiple quantum emitters.

This work is supported by National Natural Science Foundation of China (Grant No. 11875011); Educational Commission of Jilin Province of China (Grant No. JJKH20190266KJ); the China Scholarship Council (CSC) Grant No. 201806625045 (Z. W.); Austrian Science Fund (Grant No. P31701-N27); DK CoQuS Grant No. W1210 (T. J.), and an ESQ fellowship (P. K.) and Discovery Grant (P.R.) of the Austrian Academy of Sciences (ÖAW).

*Corresponding author.

wangzh761@nenu.edu.cn

${ }^{\dagger}$ Present address: Department of Physics and SUPA, University of Strathclyde, Glasgow G4 0NG, United Kingdom.

[1] R. H. Dicke, Coherence in spontaneous radiation processes, Phys. Rev. 93, 99 (1954).
[2] M. Gross and S. Haroche, Superradiance: An essay on the theory of collective spontaneous emission, Phys. Rep. 93, 301 (1982).

[3] S. Hughes, Enhanced single-photon emission from quantum dots in photonic crystal waveguides and nanocavities, Opt. Lett. 29, 2659 (2004).

[4] J.-T. Shen and S. Fan, Coherent Single Photon Transport in a One-Dimensional Waveguide Coupled with Superconducting Quantum Bits, Phys. Rev. Lett. 95, 213001 (2005).

[5] D. E. Chang, A. S. Sørensen, E. A. Demler, and M. D. Lukin, A single-photon transistor using nanoscale surface plasmons, Nat. Phys. 3, 807 (2007).

[6] L. Zhou, Z. R. Gong, Y.-x. Liu, C. P. Sun, and F. Nori, Controllable Scattering of a Single Photon Inside a OneDimensional Resonator Waveguide, Phys. Rev. Lett. 101, 100501 (2008).

[7] P. Longo, P. Schmitteckert, and K. Busch, Few-Photon Transport in Low-Dimensional Systems: Interaction-Induced Radiation Trapping, Phys. Rev. Lett. 104, 023602 (2010).

[8] H. Zheng, D. J. Gauthier, and H. U. Baranger, Waveguide QED: Many-body bound-state effects in coherent and Fockstate scattering from a two-level system, Phys. Rev. A 82, 063816 (2010).

[9] F. Lombardo, F. Ciccarello, and G. M. Palma, Photon localization versus population trapping in a coupled-cavity array, Phys. Rev. A 89, 053826 (2014).

[10] E. Shahmoon, P. Grisins, H. P. Stimming, I. Mazets, and G. Kurizki, Highly nonlocal optical nonlinearities in atoms trapped near a waveguide, Optica 3, 725 (2016).

[11] D. Roy. C. M. Wilson and O. Firstenberg, Colloquium: Strongly interacting photons in one-dimensional continuum, Rev. Mod. Phys. 89, 021001 (2017).

[12] D. Reitz, C. Sayrin, R. Mitsch, P. Schneeweiss, and A. Rauschenbeutel, Coherence Properties of Nanofiber-Trapped Cesium Atoms, Phys. Rev. Lett. 110, 243603 (2013).

[13] R. Yalla, M. Sadgrove, K. P. Nayak, and K. Hakuta, Cavity Quantum Electrodynamics on a Nanofiber Using a Composite Photonic Crystal Cavity, Phys. Rev. Lett. 113, 143601 (2014).

[14] J. D. Hood, A. Goban, A. Asenjo-Garcia, M. Lu, S.-P. Yu, D. E. Chang, and H. J. Kimble, Atom-atom interactions around the band edge of a photonic crystal waveguide, Proc. Natl. Acad. Sci. U.S.A. 113, 10507 (2016).

[15] N. V. Corzo, J. Raskop, A. Chandra, A. S. Sheremet, B. Gouraud, and J. Laurat, Waveguide-coupled single collective excitation of atomic arrays, Nature (London) 566, 359 (2019).

[16] P. Lodahl, S. Mahmoodian, and S. Stobbe, Interfacing single photons and single quantum dots with photonic nanostructures, Rev. Mod. Phys. 87, 347 (2015).

[17] O. Astafiev, A. M. Zagoskin, A. A. Abdumalikov, Yu. A. Pashkin, T. Yamamoto, K. Inomata, Y. Nakamura, and J. S. Tsai, Resonance fluorescence of a single artificial atom, Science 327, 840 (2010).

[18] I.-C. Hoi, C. M. Wilson, G. Johansson, T. Palomaki, B. Peropadre, and P. Delsing, Demonstration of a SinglePhoton Router in the Microwave Regime, Phys. Rev. Lett. 107, 073601 (2011).

[19] J. A. Mlynek, A. A. Abdumalikov, C. Eichler, and A. Wallraff, Observation of Dicke superradiance for two 
artificial atoms in a cavity with high decay rate, Nat. Commun. 5, 5186 (2014).

[20] M. Mirhosseini, E. Kim, X. Zhang, A. Sipahigil, P. B. Dieterle, A. J. Keller, A. Asenjo-Garcia, D. E. Chang, and O. Painter, Cavity quantum electrodynamics with atom-like mirrors, Nature (London) 569, 692 (2019).

[21] N. M. Sundaresan, R. Lundgren, G. Zhu, A. V. Gorshkov, and A. A. Houck, Interacting Qubit-Photon Bound States with Superconducting Circuits, Phys. Rev. X 9, 011021 (2019).

[22] D. E. Chang, J. I. Cirac, and H. J. Kimble, Self-Organization of Atoms Along a Nanophotonic Waveguide, Phys. Rev. Lett. 110, 113606 (2013).

[23] T. Grießer and H. Ritsch, Light-Induced Crystallization of Cold Atoms in a 1D Optical Trap, Phys. Rev. Lett. 111, 055702 (2013).

[24] D. Dzsotjan, A. S. Sørensen, and M. Fleischhauer, Quantum emitters coupled to surface plasmons of a nanowire: A Green's function approach, Phys. Rev. B 82, 075427 (2010).

[25] A. Gonzalez-Tudela, D. Martin-Cano, E. Moreno, L. Martin-Moreno, C. Tejedor, and F. J. Garcia-Vidal, Entanglement of Two Qubits Mediated by One-Dimensional Plasmonic Waveguides, Phys. Rev. Lett. 106, 020501 (2011).

[26] K. Stannigel, P. Rabl, and P. Zoller, Driven-dissipative preparation of entangled states in cascaded quantum-optical networks, New J. Phys. 14, 063014 (2012).

[27] H. Zheng and H. U. Baranger, Persistent Quantum Beats and Long-Distance Entanglement from WaveguideMediated Interactions, Phys. Rev. Lett. 110, 113601 (2013).

[28] E. Shahmoon and G. Kurizki, Nonradiative interaction and entanglement between distant atoms, Phys. Rev. A 87, 033831 (2013).

[29] P. Facchi, M. S. Kim, S. Pascazio, F. V. Pepe, D. Pomarico, and T. Tufarelli, Bound states and entanglement generation in waveguide quantum electrodynamics, Phys. Rev. A 94, 043839 (2016).

[30] S. Mahmoodian, P. Lodahl, and A. S. Sørensen, Quantum Networks with Chiral Light-Matter Interaction in Waveguides, Phys. Rev. Lett. 117, 240501 (2016).

[31] A. Asenjo-Garcia, M. Moreno-Cardoner, A. Albrecht, H. J. Kimble, and D. E. Chang, Exponential Improvement in Photon Storage Fidelities Using Subradiance and "Selective Radiance" in Atomic Arrays, Phys. Rev. X 7, 031024 (2017).

[32] M. J. Hartmann, F. G. S. L. Brandão, and M. B. Plenio, Strongly interacting polaritons in coupled arrays of cavities, Nat. Phys. 2, 849 (2006).

[33] A. D. Greentree, C. Tahan, J. H. Cole, and C. L. Hollenberg, Quantum phase transitions of light, Nat. Phys. 2, 856 (2006).

[34] D. G. Angelakis, M. F. Santos, and S. Bose, Photon-blockadeinduced Mott transitions and XY spin models in coupled cavity arrays, Phys. Rev. A 76, 031805(R) (2007).

[35] I. Carusotto and C. Ciuti, Quantum fluids of light, Rev. Mod. Phys. 85, 299 (2013).

[36] D. G. Angelakis, Quantum Simulations with Photons and Polaritons (Springer, New York, 2017).

[37] A. A. Houck, H. E. Türeci, and J. Koch, On-chip quantum simulation with superconducting circuits, Nat. Phys. 8, 292 (2012).
[38] A. Degiron and D. R. Smith, Nonlinear long-range plasmonic waveguides, Phys. Rev. A 82, 033812 (2010).

[39] M. Kauranen and A. V. Zayats, Nonlinear plasmonics, Nat. Photonics 6, 737 (2012).

[40] S. Haroche and J.-M. Raimond, Exploring the Quantum: Atoms, Cavities, and Photons (Oxford University Press, New York, 2006).

[41] A. T. Black, J. K. Thompson, and V. Vuletić, On-Demand Superradiant Conversion of Atomic Spin Gratings Into Single Photons with High Efficiency, Phys. Rev. Lett. 95, 133601 (2005).

[42] E. Akkermans, A. Gero, and R. Kaiser, Photon Localization and Dicke Superradiance in Atomic Gases, Phys. Rev. Lett. 101, 103602 (2008).

[43] M. O. Scully, Collective Lamb Shift in Single Photon Dicke Superradiance, Phys. Rev. Lett. 102, 143601 (2009).

[44] T. Bienaimé, N. Piovella, and R. Kaiser, Controlled Dicke Subradiance from a Large Cloud of Two-Level Systems, Phys. Rev. Lett. 108, 123602 (2012).

[45] L. Ostermann, H. Ritsch, and C. Genes, Protected State Enhanced Quantum Metrology with Interacting Two-Level Ensembles, Phys. Rev. Lett. 111, 123601 (2013).

[46] M. O. Scully, Single Photon Subradiance: Quantum Control of Spontaneous Emission and Ultrafast Readout, Phys. Rev. Lett. 115, 243602 (2015).

[47] A. Angerer et al., Superradiant emission from colour centres in diamond, Nat. Phys. 14, 1168 (2018).

[48] Y.-X. Zhang and K. Mølmer, Theory of Subradiant States of a One-Dimensional Two-Level Atom Chain, Phys. Rev. Lett. 122, 203605 (2019).

[49] Y. Ke, A. V. Poshakinskiy, C. Lee, Y. S. Kivshar, and A. N. Poddubny, Inelastic Scattering of Photon Pairs in Qubit Arrays with Subradiant States, Phys. Rev. Lett. 123, 253601 (2019).

[50] See Supplemental Material at http://link.aps.org/ supplemental/10.1103/PhysRevLett.124.213601, which includes the additional Ref. [51], for a discussion of the nature of the bound states, derivation of the master equation used, more details about the subradiant states, and mean-field results about the supercorrelated decay process.

[51] Z. Leghtas et al., Confining the state of light to a quantum manifold by engineered two-photon loss, Science 347, 853 (2015).

[52] R. Piil and K. Mølmer, Tunneling couplings in discrete lattices, single-particle band structure, and eigenstates of interacting atom pairs, Phys. Rev. A 76, 023607 (2007).

[53] M. Valiente and D. Petrosyan, Two-particle states in the Hubbard model, J. Phys. B 41, 161002 (2008).

[54] K. Winkler, G. Thalhammer, F. Lang, R. Grimm, J. Hecker Denschlag, A. J. Daley, A. Kantian, H. P. Büchler, and P. Zoller, Repulsively bound atom pairs in an optical lattice, Nature (London) 441, 853 (2006).

[55] G. Calajó, F. Ciccarello, D. Chang, and P. Rabl, Atom-field dressed states in slow-light waveguide QED, Phys. Rev. A 93, 033833 (2016).

[56] H. Breuer and F. Petruccione, The Theory of Open Quantum Systems (Oxford University Press, New York, 2002).

[57] D. E. Chang, L. Jiang, A. V. Gorshkov, and H. J. Kimble, Cavity QED with atomic mirrors, New J. Phys. 14, 063003 (2012). 
[58] A. Gonzales-Tudela and D. Porras, Mesoscopic Entanglement Induced by Spontaneous Emission in SolidState Quantum Optics, Phys. Rev. Lett. 110, 080502 (2013).

[59] A. J. Daley, Quantum trajectories and open many-body quantum systems, Adv. Phys. 63, 77 (2014).

[60] M. Leib, F. Deppe, A. Marx, R. Gross, and M. J. Hartmann, Networks of nonlinear superconducting transmission line resonators, New J. Phys. 14, 075024 (2012).

[61] S. Hacohen-Gourgy, V. V. Ramasesh, C. D. Grandi, I. Siddiqi, and S. M. Girvin, Cooling and Autonomous Feedback in a Bose-Hubbard Chain with Attractive Interactions, Phys. Rev. Lett. 115, 240501 (2015).

[62] P. Roushan et al., Spectroscopic signatures of localization with interacting photons in superconducting qubits, Science 358, 1175 (2017).

[63] R. Ma, B. Saxberg, C. Owens, N. Leung, Y. Lu, J. Simon, and D. I. Schuster, A dissipatively stabilized Mott insulator of photons, Nature (London) 566, 51 (2019).
[64] M. Kjaergaard, M. E. Schwartz, J. Braumüller, P. Krantz, J. I.-J. Wang, S. Gustavsson, and W. D. Oliver, Superconducting qubits: Current state of play, Annu. Rev. Condens. Matter Phys. 11, 369 (2020).

[65] D. Petrosyan and M. Fleischhauer, Quantum Information Processing with Single Photons and Atomic Ensembles in Microwave Coplanar Waveguide Resonators, Phys. Rev. Lett. 100, 170501 (2008).

[66] S. D. Hogan, J. A. Agner, F. Merkt, T. Thiele, S. Filipp, and A. Wallraff, Driving Rydberg-Rydberg Transitions from a Coplanar Microwave Waveguide, Phys. Rev. Lett. 108, 063004 (2012).

[67] M. A. Beck, J. A. Isaacs, D. Booth, J. D. Pritchard, M. Saffman, and R. McDermott, Optimized coplanar waveguide resonators for a superconductor-atom interface, Appl. Phys. Lett. 109, 092602 (2016).

[68] G. Calajó and P. Rabl, Strong coupling between atoms and slow-light Cherenkov photons, Phys. Rev. A 95, 043824 (2017). 